\title{
The clinical analysis of pulmonary lymphoepithelioma-like carcinoma with epithelioid granuloma
}

\author{
Guoying Gao ${ }^{1 \#}$, Chengzhi Zhou ${ }^{1 \#}$, Guofeng Wu ${ }^{3 \#}$, Weijie Guan ${ }^{1}$, Guosheng Deng ${ }^{2}$, Zheng Zhu ${ }^{1}$, \\ Xinqing Lin ${ }^{1}$, Xiaohong Xie ${ }^{1}$, Zhanhong Xie ${ }^{1}$, Jiexia Zhang ${ }^{1}$, Ming Ouyang ${ }^{1}$, Nanshan Zhong ${ }^{1}$, \\ Shiyue Li $^{1}$, Yinyin Qin ${ }^{1,2}$
}

${ }^{1}$ State Key Laboratory of Respiratory Disease, National Clinical Research Center for Respiratory Disease, Guangzhou Institute of Respiratory Health, The First Affiliated Hospital of Guangzhou Medical University, Guangzhou 510120, China; ${ }^{2}$ Shunde Affiliated Hospital of Guangzhou Medical University, Foshan 528315, China; ${ }^{3}$ The Third Affiliated Hospital of Guangzhou Medical University Li-Wan Hospital, Guangzhou 510170, China Contributions: (I) Conception and design: G Gao, C Zhou, G Wu, Y Qin; (II) Administrative support: N Zhong, S Li; (III) Provision of study materials or patients: X Lin, X Xie, Z Xie, J Zhang, O Ming; (IV) Collection and assembly of data: G Gao, C Zhou, G Wu, Y Qin, W Guan, G Deng, Z Zhu; (V) Data analysis and interpretation: G Gao, C Zhou, G Wu, Y Qin; (VI) Manuscript writing: All authors; (VII) Final approval of manuscript: All authors.

\#These authors contributed equally to this work.

Correspondence to: Yinyin Qin. State Key Laboratory of Respiratory Disease, National Clinical Research Center for Respiratory Disease, Guangzhou Institute of Respiratory Health, The First Affiliated Hospital of Guangzhou Medical University, Guangzhou 510120, China. Email: 13610047638@163.com.

Background: Pulmonary lymphoepithelioma-like carcinoma (PLELC) is a rare subtype of non-small cell lung cancer associated with Epstein-bar virus (EBV) infection. Epithelioid granuloma (EG) has been more scarcely reported and frequently misdiagnosed.

Methods: Data were collected from January 2013 to October 2019. Of 227 patients diagnosed as having PLELC, 22 patients had EG. We analyzed their clinical features, pathological characteristics and treatment and a comparison between PLELC patients complicated with or without EG was made.

Results: Twenty-two patients had complicated with EG (9.6\%). The median age was 50 years $(38-$ 67 years). There were more females than males (1.4:1). Most patients were at early stage (68.2\%) with nonspecific manifestations and lack of Rich-Lewis phenomenon. Compared with the 205 patients complicated without $\mathrm{EG}$, there were no significant difference among age $(\mathrm{t}=0.938, \mathrm{P}=0.349)$, gender $\left(\chi^{2}=0.898, \mathrm{P}=0.343\right)$, initial symptoms $\left(\chi^{2}=2.684, \mathrm{P}=0.443\right)$, smoking status $\left(\chi^{2}=0.210, \mathrm{P}=0.647\right)$, diameter of tumor $(\mathrm{t}=0.993, \mathrm{P}=0.332)$ and performance status $(\mathrm{H}=0.971, \mathrm{P}=0.615)$. EG was often located inside or adjacent to the tumor (71.4\%). Specific staining was negative, whereas in situ hybridization staining of EBV-encoded RNA was consistently positive. Most patients complicated with EG received multimodality therapy including surgery, neo-adjuvant/adjuvant chemotherapy or palliative chemotherapy and none of them received anti-TB therapy. Compared with the 205 patients complicated without EG, there were no significant difference among tumor stage, DFS (median, not reached, $\mathrm{P}=0.914$ ), PFS (median, 12.3 months, $\mathrm{P}=0.848$ ), OS (median, not reached, $\mathrm{P}=0.737$ ) and treatment including anti-tumor therapy and anti-TB therapy. During follow-up duration for 14.6 months (range, 2.1-94.7 months), none of the patients had occurrence, progression or relapse of tuberculosis, regardless whether anti-tuberculosis therapy was initiated. Conclusions: PLELC complicated with EG was lack of Rich-Lewis phenomenon and specific clinical characteristics compared with those without EG. EG might be caused by immunological hypersensitivity to tumor cells or EBV infection but not pulmonary tuberculosis. PLELC complicated with EG could be treated with chemotherapy and surgery. However, anti-tuberculosis therapy was unnecessary. 


\begin{abstract}
Keywords: Epithelioid granuloma (EG); lung cancer; pulmonary lymphoepithelioma-like carcinoma (PLELC);
\end{abstract} tuberculosis; treatment

Submitted Jul 05, 2019. Accepted for publication Nov 28, 2019.

doi: $10.21037 /$ tcr.2019.12.87

View this article at: http://dx.doi.org/10.21037/tcr.2019.12.87

\section{Introduction}

Epithelioid granuloma (EG) is a disease in which macrophages, epithelioid cells or multicellular giant cells clustered and proliferated, forming a chronic specific inflammatory response. EG is characterized by caseous necrosis, proliferation of langerhan's cell, epithelioid cells and lymphocytes, and (or) fibrous cell hyperplasia. EG could be seen in infectious diseases, vasculitis, immunological disorders, leucocyte oxidase defect, hypersensitivity, chemical-induced injury and neoplasia (1). The EG in tumors has been known as the 'sarcomatoid reaction', which was thought to be caused by immunological hypersensitivity to antigens of the tumor cells. Lung cancer including squamous cell carcinoma, adenocarcinoma, large cell carcinoma complicated with EG has been rarely reported (2-4). Pulmonary lymphoepithelioma-like carcinoma (PLELC), a rare subtype of primary lung cancer, has been removed from the subtype of large-cell lung cancer to nonsmall cell lung cancer according to The World Health Organization Classification of Lung Tumors in 2015 (5). It represented approximately $0.92 \%$ of lung cancer (6), and $0.4 \%$ of non-small cell lung cancer (7). Over the past decades, there was increasing evidence that PLELC, resembled as nasopharyngeal carcinoma but clearly different from other lung cancers, was associated with EBV infection (8-10) and had a better prognosis than other lung cancers (11-13). The complication with EG is more scarcely seen. Moreover, less is known about the etiology, clinical manifestation and treatment of PLELC with EG. Hence, misdiagnosis and missed diagnosis were prone to take place, especially for the differentiation with tuberculosis (TB) since the insufficient understanding for EG. Here, we presented the clinical data of 22 patients with PLELC complicated with EG who were recruited from The First Affiliated Hospital of Guangzhou Medical University, and a comparison with the PLELC patients complicated without EG was also made from the same population.

\section{Methods}

\section{Patients}

We enrolled 22 patients complicated with EG, from 227 patients diagnosed as having PLELC in The First Affiliated Hospital of Guangzhou Medical University between January 2013 and October 2019. Patients were diagnosed as having PLELC complicated with EG occurring in the lung tissue or lymph nodes by senior pathologists. Patients with completed clinical data were included. The primary tumors in extra-pulmonary or nasopharyngeal carcinoma metastasis to intra-pulmonary were excluded.

\section{Study design and statistical analysis}

We retrospectively analyzed the demographic data, clinical characteristics, pathological features, tumor stage, treatment, tumor size, location of tumor and presence of EG, and follow up based on clinical data or telephone visit. Then a comparison between PLELC patients complicated with or without EG was made from the same population. Pathologic staging was performed according to the Eighth Edition Lung Cancer Stage Classification(14).

The follow-up duration was defined as the period from the date of diagnosis to the date of death for any cause or lost to follow-up. Patients were followed up until October $31^{\text {st }}, 2019$. The duration of progression-free survival (PFS) was defined as the period from the start of systematic therapy to the first date of disease progression or death. The duration of disease-free survival (DFS) was defined as the period from the initiation of systematic therapy to the date when disease relapsed. The overall survival (OS) duration was defined as the period from the time of diagnosis to the time of death for any cause or was censored at the time to the last follow-up.

Software SPSS16.0 (IBM, SPSS Armonk, NY, USA) was used for the statistical analysis. Where applicable, the results were compared using two-independent sample $t$-test, 
Pearson chi square, Fisher's exact test or Wilcoxon tests. Survival analysis were estimated using Kaplan-Meier curve with log-rank test. A 2-sided $\mathrm{P}$ value of less than 0.05 was deemed statistically significant.

\section{Results}

\section{Clinical features}

We analyzed 227 patients with PLELC. Among the 22 patients complicated with EG, $86.3 \%$ of them came from Guangdong Province, China. The median age was 50 years (38-67 years), and $18(81.8 \%)$ were age less than 60 years. Nine $(40.9 \%)$ were male, with a male to female ratio of $1: 1.4$, six $(27.3 \%)$ were smokers, two $(9.1 \%)$ with a history of $\mathrm{TB}$, and the initial symptoms were including cough or productive cough (54.6\%), hemoptysis (9.1\%), or even lack of symptom $(22.7 \%)$ but accidentally diagnosed by routine examination. None had Rich-Lewis phenomenon such as hypothermia, night sweats or weak. The median diameter of the tumor was $4.2 \mathrm{~cm}(1.1-8.4 \mathrm{~cm})$, and the diameter in $13(59.1 \%)$ patients was larger than $3.5 \mathrm{~cm}$. Radiologically, 10 (45.5\%) patients had central soft lesions surrounded by vessels and bronchi $(8 / 80 \%)$ and $12(54.5 \%)$ patients had peripheral nodule with shallow lobulation $(8 / 66.7 \%)$ or speculation $(7 / 58.3 \%), 2$ patients presented with cavitation, but calcification or fibrosis was rarely seen. Tumor was located in the lower lobe in $15(68.2 \%)$ patients. Seven patients tested the acid-fast staining of sputum smear and was consistently negative, two patients tested borderline positive to TB antibody. Ten patients tested negative to quantitative quantification of TB-RNA and two patients tested positive to T-spot test. Compared with the 205 patients complicated without EG, there were no significant difference among age $(t=0.938$, $\mathrm{P}=0.349)$, gender $\left(\chi^{2}=0.898, \mathrm{P}=0.343\right)$, initial symptoms $\left(\chi^{2}=2.684, \mathrm{P}=0.443\right)$, smoking status $\left(\chi^{2}=0.210, \mathrm{P}=0.647\right)$, diameter of tumor $(\mathrm{t}=0.993, \mathrm{P}=0.332)$ and performance status $(\mathrm{H}=0.971, \mathrm{P}=0.615)$ (Table 1).

\section{Patbological characteristics}

\section{Histopathologic manifestation}

EG was located inside or adjacent to the tumor (81.8\%), or within lymph nodes (18.2\%) (Figures 1,2). It microscopically manifested as the undifferentiated tumor cells with unclear boundaries. The tumor cells were circular or oval with large and eosinophilic nuclei. In addition, the cells were rich in cytoplasm, had an irregular nuclear, which presented with the vacuolar chromatin, clustered like syncytium and aligned in a solid nested bulk. The surrounding stroma was infiltrated by multiple lymphocytes and plasma cells (Figure 3). Apart from these characteristics, the epithelioid cells, multicellular giant cells, Langerhans giant cells with or without central caseous necrosis could be observed inside or adjacent to the tumor (Figures 1,2). The nests of epithelioid cell and (or) multicellular giant cells without necrosis was observed in 16 patients, while necrosis was presented in 6 patients, including 3 patients with the typical pathologic features of tuberculosis, which manifested as caseous necrosis surrounded by epithelioid cells and Langerhans' giant cells.

\section{Immunohistochemistry and special staining}

All 22 patients presented with a high proportion of expression of CK (100.0\%/10), CK5/6 (100.0\%/12) and P63 $(100.0 \% / 14)$ and low proportion of expression of CK7 (0.0\%/4), TTF-1 (9.0\%/11) with positive staining for in situ hybridization of Epstein-Bar Virus-related RNAs (EBERs) (Figure 4). Pathological specific staining of all 22 patients was consistently negative, including acid-fast staining in 17 patients (Figure 5), periodic acid-schiff staining in 12 patients, methenamine silver staining in 15 patients, elastic tissue staining in 8 patients, Gram staining in 2 cases and Giemsa staining in 2 patients.

\section{Treatment and survival}

Two hundred twenty-seven patients including 22 patients complicated with EG were followed up until October $31^{\text {st }}$ 2019 and the median follow-up duration was 14.6 months (range, $2.1-94.7$ months), with $18.9 \%$ of patients being lost to follow-up. In patients complicated with EG, most of them were at the early stage $(68.2 \%)$, including 14 patients with complete resection and 10 with adjuvant or neo-adjuvant chemotherapy, and 7 patients with advanced disease received palliative chemotherapy. Most regimens of chemotherapies were third-generation platinum-based combination with gemcitabine, paclitaxel, or pemetrexed. None of the patients was treated with anti-TB therapy but free of Rich-Lewis phenomenon associated with TB during follow-up. One of them was died in 6 months. The median OS and DFS time were not reach, the median PFS in the patient at advanced stage was 11.4 months. Compared with the 205 patients complicated without EG, there were no significant difference among tumor stage, DFS (median, not reached, $\mathrm{P}=0.914$, Figure 6), PFS (median, 12.3 months, 
Table 1 General characteristics and subgroup analysis of the patients with $(\mathrm{n}=22)$ and without $(\mathrm{n}=205)$ epithelioid granuloma

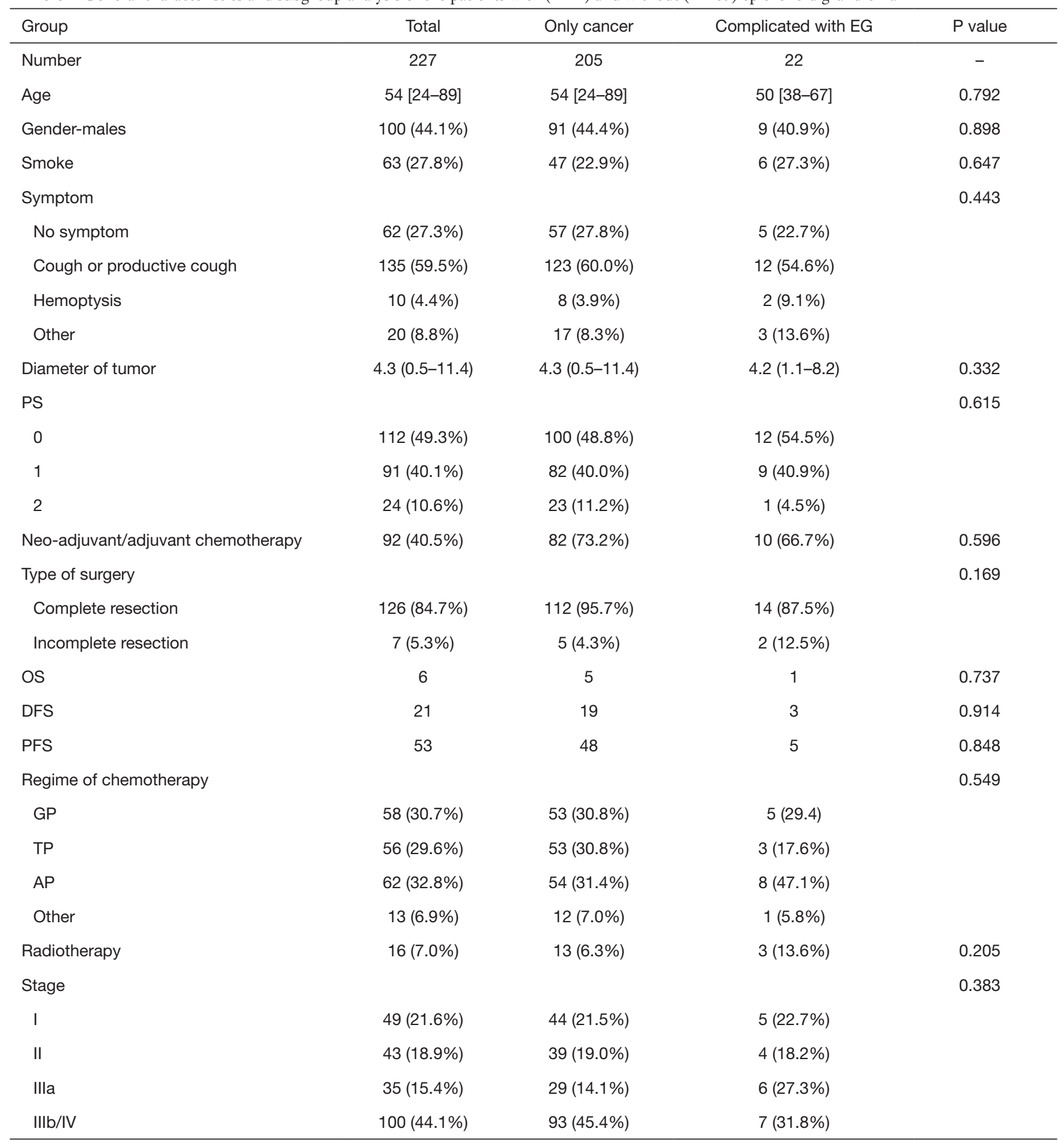

EG, epithelioid granuloma; M, male; PS, performance status; OS, overall survival; DFS, disease-free survival; PFS, progression-free survival; PP, pemetrexed plus platinum; GP, Gemcitabine plus platinum. 


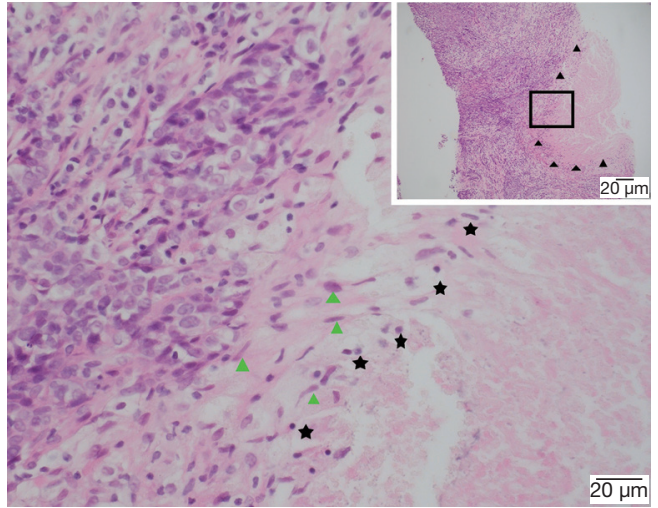

Figure $1 \mathrm{EG}$ with a typical pathologic changes like TB manifested as caseous necrosis adjacent to the tumor $(\boldsymbol{\Delta}, 100 \times)$. The EG consisted of caseous necrosis in the central ( $\star$ ), surrounded by epithelioid cells ( $\Delta)(400 x)$.

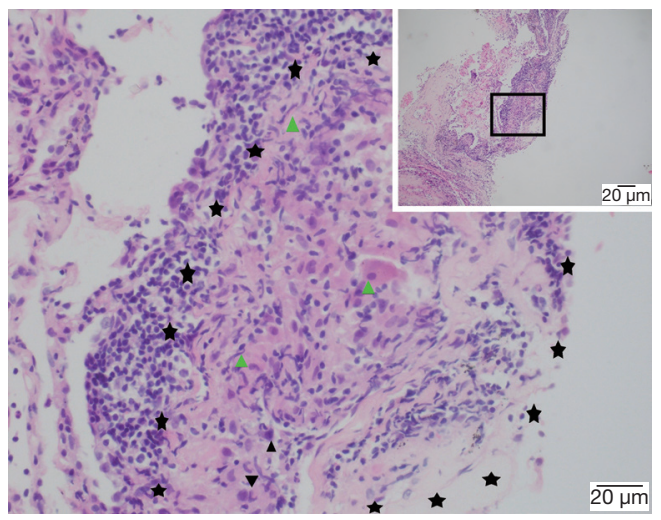

Figure $2 \mathrm{EG}$ manifestation as tuberculous granuloma beside the tumor $(100 \times)$. The EG $(\star)$ consisted of multicellular giant cells, Langhans' giant cells in the central $(\boldsymbol{\Delta})$, surrounded by epithelioid cells $(\mathbf{\Delta})$ and Large numbers of reactive lymphoplasmacytic cells $(400 \times)$.

$\mathrm{P}=0.848$, Figure 7), OS (median, not reached, $\mathrm{P}=0.737$, Figure 8) and treatment including anti-tumor therapy and anti-TB therapy. Results are summarized in Table 1.

\section{Discussion}

Primary PLELC complicated with EG has been rarely reported. To our knowledge, only 28 cases have been reported in four studies (15-18), and the 22 cases reported in this study represents the largest cohort to date. PLELC is prone to be more frequently identified in patients with

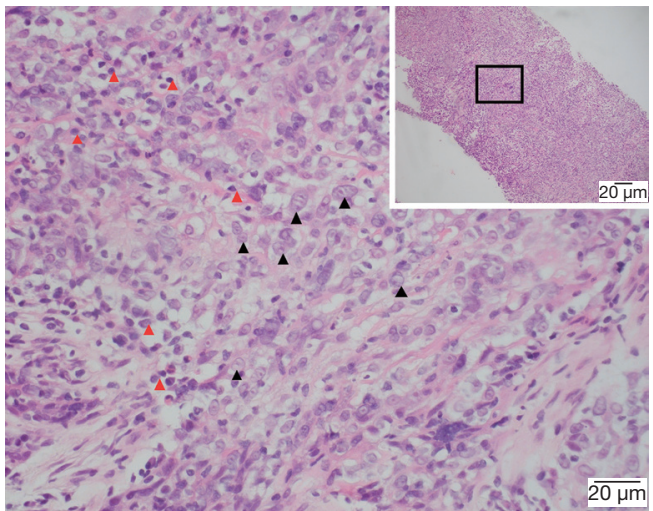

Figure 3 Histology of lymphoepithelioma-like carcinoma (100x). The tumor cell $(400 \times, \boldsymbol{\Delta})$ nuclei are round, oval or elongated with mildly irregular nuclear outline, vesicular chromatin and distinct nucleoli. Large numbers of reactive lymphoplasmacytic cells in stroma $(\boldsymbol{\Delta})$.

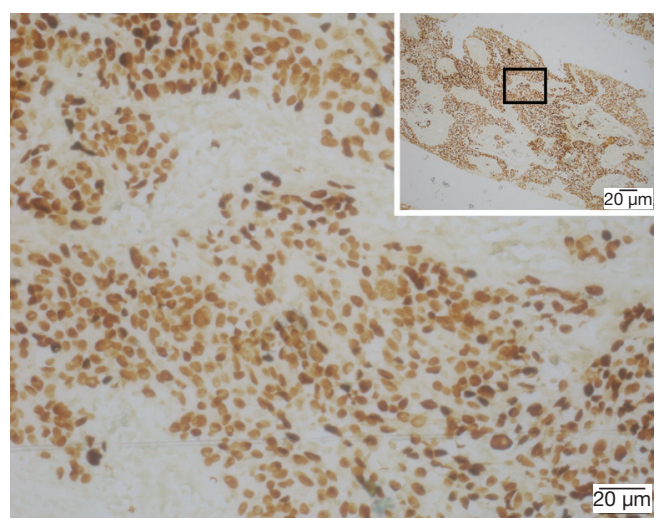

Figure 4 In EBERs (100x and 400x), showing intense signals in the nuclei of the majority of tumor cells.

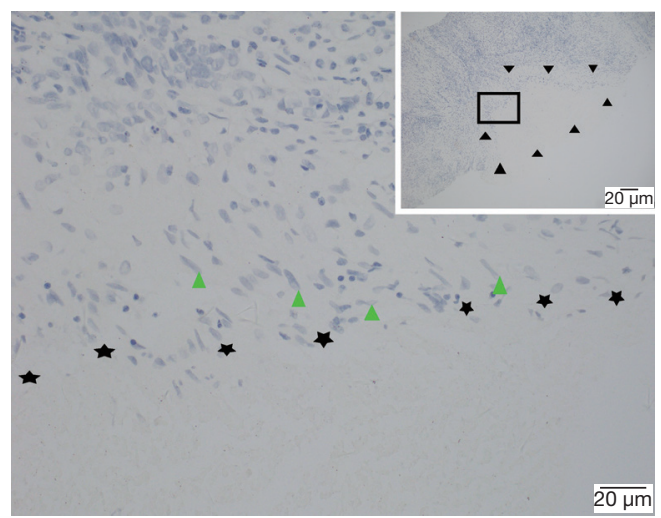

Figure 5 EG with acid fast staining negatively (100x and 400x). 


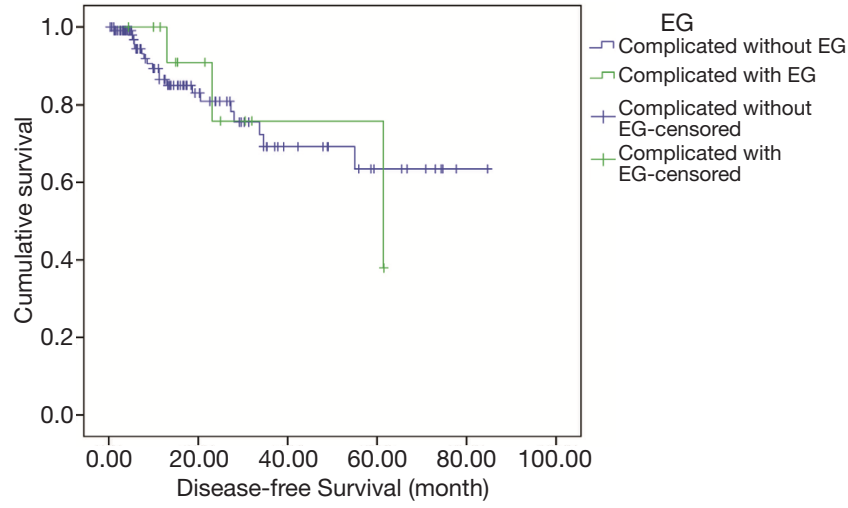

Figure 6 Comparison of the duration of disease-free survival (DFS) between patients with PLELC at early stage whether complicated with $\mathrm{EG}(\mathrm{P}=0.914)$.

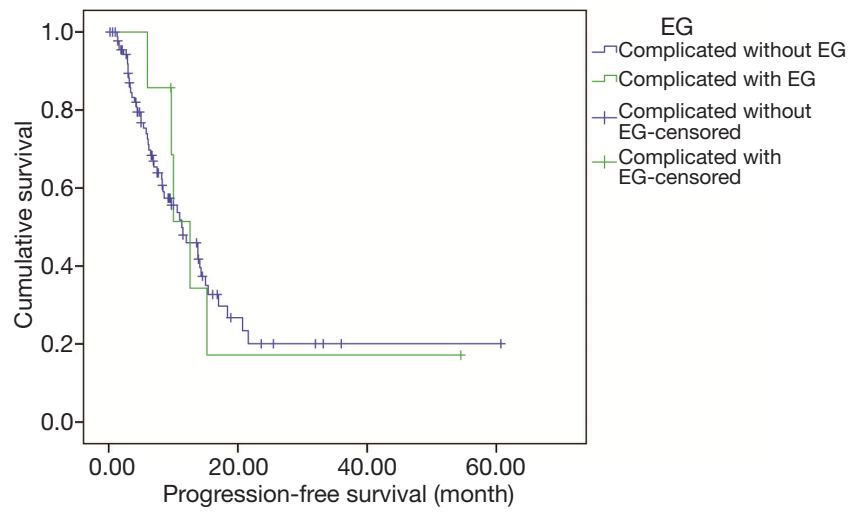

Figure 7 Comparison of the duration of progression-free survival (PFS) between patients with PLELC at advanced stage whether complicated with EG $(\mathrm{P}=0.848)$.

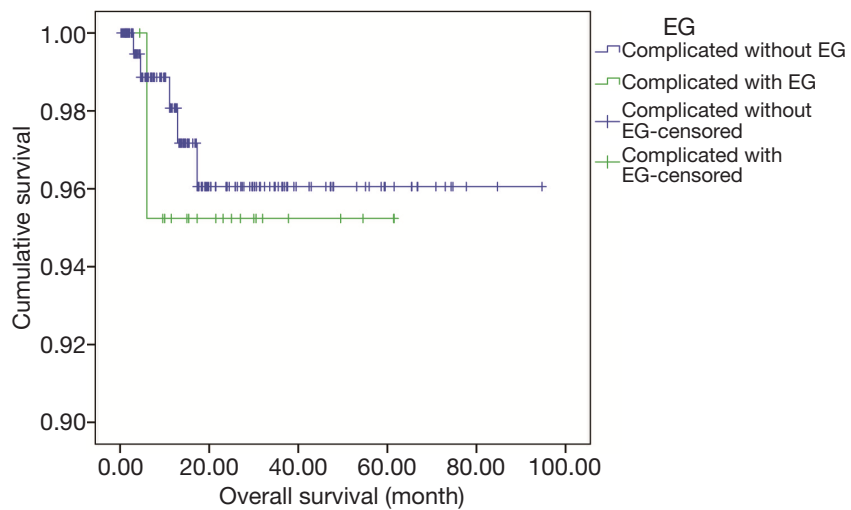

Figure 8 Comparison of the overall survival (OS) duration between patients with PLELC whether complicated with EG $(\mathrm{P}=0.737)$. certain ethnicity and regions, especially in young nonsmokers from Asian regions, such as Guangdong Province (6,19-23), Fujian province (24), Taiwan (25,26), Hong Kong (16). However, there were no significant differences between males and females. In this group, most of the patients complicated with EG came from Guangdong Province, the southern of China, the median age was 50 years (range, 38-67 years), 81.8\% of them were aged 60 years or lower with nonsmoking, which was accordance with those reported by Liang et al. $(12,21)$. The initial symptoms were including cough or productive cough, hemoptysis, or even asymptomatic but lack of Rich-Lewis phenomenon. The imaging appeared as a larger soft tissue centrally surrounded by vessels and bronchi or appeared as a peripheral nodule with unclear lobular boundaries and adjacent to the pleura $(7,12,27,28)$, which was in line with this study that lesions always presented as larger tumor size centrally or peripherally in the lower lobe but seldom accompanied with cavitation, calcification or fibrosis. Compared with the 205 patients complicated without EG, there were no significant difference among age, gender, initial symptoms, smoking status, diameter of tumor and performance status. Hence, it indicated that patients complicated with EG had nonspecific clinical features, which mainly depended on characteristics of tumor.

Han et al. (17) reported that EG was located in the stroma, and caseous necrosis was observed in the center of tuberculoid nodules. Meanwhile, TB consists of eosinophilic caseous necrosis located in the multicenter with different diameters, surrounded by epithelioid cells, multinucleated giant cells, and less frequently seen were the fibrocytes. The granuloma was frequently lumped together and distributed abnormally. In some of these patients, epithelioid cells and multinucleated giant cell without caseous necrosis was the sole manifestation among atypical cases (29-32). The positive rate of the acid-fast staining in TB was $62.96 \%$ (33). In this study, EG was more often identified to be situated inside or adjacent to the tumor rather than within the lymph nodes, which consisted of the epithelioid cells, multicellular giant cells, and (or) caseous necrosis occasionally occur. However, the specific staining including acid-fast staining, periodic acid-schiff stain, methenamine silver stain, elastic tissue stain was consistently negative. Furthermore, the pathologic morphology of the tumor cells in this study was consistent with the findings in previous reports $(12,18,21,34)$, it was higher expression of CK, CK5/6 and P63 but minor expression of CK7, TTF-1 and all the patients had notably positive staining of EBER. Therefore, 
it showed that PLELC complicated with EG had similar pathologic morphology of the tumor with patients without $\mathrm{EG}$, beside the EG, then we speculated that the EG in the patients with PLECL might be unrelated to TB.

Han et al. (17) considered that the tuberculoid granuloma scattered in the interstitium was associated with the tumor. The macrophages swallowed the necrotic tissue or lipid from the tumor, and formed the epithelioid cells, which usually do not develop into caseous necrosis that is different from tubercle. Some studies reported LELC complicated with EG that was derived from extra-pulmonary such as nasopharynx (35), breast (36), stomach (37), thymus (38). Torre et al. (35) reported nasopharyngeal LELC accompanied with multicellular giant cells pathologically, and the particular granulomatous reaction was thought to be due to the host's defense to the tumor cells, but also to the reaction to the viral antigens, However, Shet et al. (36) reported the LELC in the breast but as the EBERs were not detected in the tumors. Hence, they reported that these granulomas could represent the host's immune response to tumor antigens and was unrelated to viral infection. In this study, EBERs tested consistently positive and fastacid staining was all negative. Therefore, we proposed that PLELC was closely related to EBV infection, and the pathological changes of EG might be related to EBV infection and the host's defense to the tumor cells, but not necessarily to mycobacterium tuberculosis infection.

Until now, due to its limited numbers, the treatment related to whether is it necessary to receive anti-TB therapy and whether is it could to receive anti-tumor therapy for PLELC patients complicated with EG has not been reported. Yakar et al. (3) reported 48 postoperative patients with NSCLC complicated with necrotizing granulomatous, which did not receive anti-TB therapy and had non-TB related symptoms occurring during the 2-year follow-up period. Therefore, it was suggested that necrotizing granulomatous detected in surgical resection did not require anti-TB therapy, despite the presence of specific imaging manifestations such as cavity, calcification. In another study (2), there were 19 patients with lung cancer complicated with granuloma from Singapore, a TB-epidemic areas, including 11 of those who underwent adjuvant or neo-adjuvant chemotherapy before or after surgery, and 8 patients received surgery. And TB was not reported during 1.5 years of follow-up. Hence, the authors provided that this might be possibly due to either TB or previous non-tuberculous mycobacteria infection or sarcomatoid reaction to cancer, and these patients might be managed as lung cancer patients without coexisting granulomatous inflammation, and chemotherapy was safe. In our group, most patients complicated with EG received multimodality therapy including surgery, neo-adjuvant/ adjuvant chemotherapy or palliative chemotherapy and none of them received anti-TB therapy. During the followup time for 14.6 months, all were free from TB infections. Finally, there were no significant difference in OS (median, not reached, $\mathrm{P}=0.737$ ) at overall patients, DFS (median, not reached, $\mathrm{P}=0.914)$ at the early stages and PFS (11.3 vs. $12.3, \mathrm{P}=0.848)$ at the advanced stages between patients complicated with or without EG, which indicated that EG was not the prognostic factors for patients with PLELC. Therefore, we deemed that anti-TB therapy would not be necessary. Alternatively, we recommend that these patients should receive multimodality therapy including chemotherapy, radiotherapy and surgical treatment if the active stage of TB had been carefully excluded.

Our study was novel in some aspects. First, we sampled a large sample size of PLELC patients complicated with EG. Second, this study made a comparison between patients with PLELC complicated with or without EG. Third, our study had provided that EG was not the prognostic factors for patients with PLELC.

There are some limitations. This was a retrospective study, and the duration of follow-up was not sufficiently long. In addition, the mechanism of pathogenesis for EG was still unknown.

\section{Conclusions}

In conclusion, PLELC complicated with EG was lack of Rich-Lewis phenomenon and specific clinical characteristics compared with those without EG. EG might be caused by immunological hypersensitivity to tumor cells or EBV infection but not pulmonary tuberculosis. PLELC complicated with EG could be treated with chemotherapy and surgery. However, anti-tuberculosis therapy was unnecessary.

\section{Acknowledgments}

Funding: This work was supported by National Natural Science Foundation of China (81670036), Mandatory subject project of medical research fund of Guangdong province(C2019029)/Self-funded science and technology plan project of Guangdong department of science and technology (2019ZC0029), Guangdong High Level 
University Clinical Cultivation Project (201721020), State Key Laboratory of Respiratory Disease-The Independent project (SKLRD-MS-201905, SKLRD-QN 201720), characteristic innovation projects of universities in Guangdong province (2018KTSCX181), State Key Laboratory of Respiratory Disease-The open project (SKLRD-OP-2018011).

\section{Footnote}

Conflicts of Interest: All authors have completed the ICMJE uniform disclosure form (available at http://dx.doi. org/10.21037/tcr.2019.12.87). The authors have no conflicts of interest to declare.

Ethical Statement: The authors are accountable for all aspects of the work in ensuring that questions related to the accuracy or integrity of any part of the work are appropriately investigated and resolved. The study was conducted in accordance with the Declaration of Helsinki (as revised in 2013). This study was conducted with approval from the Ethics Committee of The First Affiliated Hospital of Guangzhou Medical University (No. 2019-K-28-201906-10) and written informed consent was obtained from all patients.

Open Access Statement: This is an Open Access article distributed in accordance with the Creative Commons Attribution-NonCommercial-NoDerivs 4.0 International License (CC BY-NC-ND 4.0), which permits the noncommercial replication and distribution of the article with the strict proviso that no changes or edits are made and the original work is properly cited (including links to both the formal publication through the relevant DOI and the license). See: https://creativecommons.org/licenses/by-nc-nd/4.0/.

\section{References}

1. Jiang XQ, Lu ZF, Shi SS, et al. Clinicopathologic Analysis of Lung Epithelial Malignant Tumors with Epithelioid Granuloma: Report of 11 Cases. J Clin Experiment Pathol 2014;30:411-4.

2. Dagaonkar RS, Choong CV, Asmat AB, et al. Significance of Coexistent Granulomatous Inflammation and Lung Cancer. J Clin Pathol 2017;70:337-41.

3. Yakar F, Yakar A, Büyükpınarbaşı1ı N, et al. Does Every Necrotizing Granulomatous Inflammation Identified by NSCLC Resection Material Require Treatment? Med Sci
Monit 2016;22:1218-22.

4. Tajima S, Koda K. Granulomatous Inflammation of Pulmonary Squamous Cell Carcinoma: a rare phenomenon. Int J Clin Exp Pathol 2015;8:7547-52.

5. Travis WD, Brambilla E, Nicholson AG, et al. The 2015 World Health Organization Classification of Lung Tumors: Impact of Genetic, Clinical and Radiologic Advances Since the 2004 Classification. J Thorac Oncol 2015;10:1243-60.

6. Lin Z, Situ D, Chang X, et al. Surgical treatment for primary pulmonary lymphoepithelioma-like carcinoma. Interact Cardiovasc Thorac Surg 2016;23:41-6.

7. Mo Y, Shen J, Zhang Y, et al. Primary Lymphoepitheliomalike Carcinoma of the Lung Distinct Computed Tomography Features and Associated Clinical Outcomes. J Thorac Imaging 2014;29:246-51.

8. Yang H, Lin Y, Liang Y, et al. Treatment of Lung Carcinosarcoma and Other Rare Histologic Subtypes of Non-small Cell Lung Cancer. Curr Treat Options Oncol 2017;18:54.

9. Xie M, Wu X, Wang F, et al. Clinical Significance of Plasma Epstein-Barr Virus DNA in Pulmonary Lymphoepithelioma-like Carcinoma (LELC) Patients. J Thorac Oncol 2018;13:218-27.

10. Hong S, Liu D, Luo S, et al. The Genomic Landscape of Epstein-Barr Virus-associated Pulmonary Lymphoepithelioma-like Carcinoma. Nat Commun 2019;10:3108.

11. Lin Z, Fu S, Zhou Y, et al. First-line Platinum-based Chemotherapy and Survival Outcomes in Locally Advanced or Metastatic Pulmonary Lymphoepitheliomalike Carcinoma. Lung Cancer 2019;137:100-7.

12. Qin Y, Gao GY, Xie XH et al. Clinical Features and Prognosis of Pulmonary Lymphoepithelioma-like Carcinoma: Summary of Eighty-five Cases. Clin Lung Cancer 2019;20:e329-37.

13. Zhou N, Lin Y, Peng X, et al. Thorough Survey and Analysis of Pulmonary Lymphoepithelioma-like Carcinoma in Macau and Multimodality Treatment for Advanced Disease. Lung Cancer 2019;138:116-23.

14. Detterbeck FC, Boffa DJ, Kim AW, et al. The Eighth Edition Lung Cancer Stage Classification. Chest 2017;151:193-203.

15. Guerrero A, Laflamme M, Agoff SN, et al. Primary Lymphoepithelioma-like Carcinoma of the lung. Can Respir J 2001;8:431-3.

16. Chan JK, Hui PK, Tsang WY, et al. Primary lymphoepithelioma-like carcinoma of the lung. A 
clinicopathologic study of 11 cases. Cancer 1995;76:413-22.

17. Han A, Xiong M, Gu Y, et al. Clinicopathologic features and prognosis of lymphoepithelioma-like carcinoma of the lung. Zhonghua Bing Li Xue Za Zhi 2001;30:328-31.

18. Tay CK, Chua YC, Takano A, et al. Primary Pulmonary Lymphoepithelioma-like Carcinoma in Singapore. Ann Thorac Med 2018;13:30-5.

19. Yu X, Wen Y, Qin R, et al. Prognosis and Distribution of Lymph Nodes Metastases in Resectable Primary Pulmonary Lymphoepithelioma-like Carcinoma: A Large Cohort from A Single Center. Thorac Cancer 2018:9:360-7.

20. Wang L, Long W, Li PF, et al. An Elevated Peripheral Blood Monocyte-to-Lymphocyte Ratio Predicts Poor Prognosis in Patients with Primary Pulmonary Lymphoepithelioma-Like Carcinoma. PLoS One 2015;10:e0126269.

21. Liang Y, Wang L, Zhu Y, et al. Primary Pulmonary Lymphoepithelioma-like Carcinoma: Fifty-two Patients with Long-term Follow-up. Cancer 2012;118:4748-58.

22. Han AJ, Xiong M, Gu YY, et al. Lymphoepitheliomalike Carcinoma of The Lung with A Better Prognosis. A clinicopathologic study of 32 cases. Am J Clin Pathol 2001;115:841-50.

23. Xie Z, Liu L, Lin X, et al. A Multicenter Analysis of Genomic Profiles and PD-L1 Expression of Primary Lymphoepithelioma-like Carcinoma of The Lung. Mod Pathol 2019. [Epub ahead of print].

24. Lin L, Lin T, Zeng B, et al. Primary Lymphoepitheliomalike Carcinoma of The Lung: An Unusual Cancer and Clinical Outcomes of 14 Patients. Oncol Lett 2017;14:3110-6.

25. Chang YL, Wu CT, Shih JY, et al. New Aspects in Clinicopathologic and Oncogene Studies of 23 Pulmonary Lymphoepithelioma-Like Carcinomas. Am J Surg Pathol 2002;26:715-23.

26. Lin CY, Chen YJ, Hsieh MH, et al. Advanced Primary Pulmonary Lymphoepithelioma-like carcinoma: Clinical Manifestations, Treatment, and Outcome. J Thorac Dis 2017;9:123-8.

27. Hoxworth JM, Hanks DK, Araoz PA, et al.

Cite this article as: Gao G, Zhou C, Wu G, Guan W, Deng G, Zhu Z, Lin X, Xie X, Xie Z, Zhang J, Ouyang M, Zhong N, Li S, Qin Y. The clinical analysis of pulmonary lymphoepitheliomalike carcinoma with epithelioid granuloma. Transl Cancer Res 2020;9(2):1023-1031. doi: 10.21037/tcr.2019.12.87
Lymphoepithelioma-like Carcinoma of The Lung: Radiologic Features of An Uncommon Primary Pulmonary Neoplasm. AJR Am J Roentgenol 2006;186:1294-9.

28. Ma H, Wu Y, Lin Y, et al. Computed Tomography Characteristics of Primary Pulmonary Lymphoepithelioma-like Carcinoma in 41 Patients. Eur J Radiol 2013;82:1343-6.

29. Li F, Chen YQ, Bao AH, et al. Clinical Analysis of Granulomatous Lung Disease: 36 Cases Report, Chin J Respirat Crit Care Med 2013;3:274-8.

30. Zhang DP, Meng FQ. Granulomatous Pulmonary Disease. Chin J Respiratory Crit Care Med 2011;10:202-5.

31. Aubry MC. Necrotizing Granulomatous Inflammation: What Does It Mean If Your Special Stains Are Negative? Mod Pathol 2012;25 Suppl 1:S31-8.

32. Zhao J, Lin XQ, Hu T. Clinical Pathological Analysis Between Sarcoidosis and Pulmonary Tuberculosis. J Clin Pulmonol 2016;(1):7-10.

33. Zhang DC, Liu B, Zhang LH, et al. The Value of Acidfast Staining in Pathological Diagnosis of Tuberculosis. Chin J Antitubercul 2014;4:274-8.

34. Jiang WY, Wang R, Pan XF, et al. Clinicopathological Features and Prognosis of Primary Pulmonary Lymphoepithelioma-like Carcinoma. J Thorac Dis 2016;8:2610-6.

35. Torre V, Cavallari V, Bucolo S, et al. Description of A Particular Case of The So-called Schmincke Lymphoepithelioma and Study of The Correlation with Epstein-Barr Virus. Acta Otorhinolaryngol Ital 2000;20:347-53.

36. Shet T, Pai T, Shetty O, et al. Lymphoepithelioma-like Carcinoma of Breast-evaluation for Epstein-Barr Virusencoded RNA, Human Papillomavirus, and Markers of Basal Cell Differentiation. Ann Diagn Pathol 2016;25:42-7.

37. Liu S, Jin L, Xu X, et al. Pathological and Computed Tomography Findings of Lymphoepithelioma-like Gastric Carcinoma with Epithelioid Granulomas: A Case Report. Oncol Lett 2013;5:549-51.

38. Tacyildiz N, Ugur H, Yavuz G, et al. The Coexistence of Thymic Carcinoma and Multiple Granulomas in A Turkish Child. Pediatr Hematol Oncol 2007;24:301-7. 